\title{
Drug use, consequences and perceived accessibility in three Nigerian universities
}

\author{
Peter Olutunde Onifade ${ }^{1}$, Edward Babatunde Somoye ${ }^{2}$, Oladotun Olorunfemi Ogunwobi ${ }^{3}$, \\ Babatunde Fadipe ${ }^{4}$, Ayodele Lucy Fela-Thomas ${ }^{2}$, Mojisola Anifat Adeniji ${ }^{2}$ \\ ${ }^{1}$ Drug Addiction Treatment, Education and Research Unit, Neuropsychiatric Hospital, Abeokuta, Nigeria \\ ${ }^{2}$ Neuropsychiatric Hospital, Abeokuta, Nigeria \\ ${ }^{3}$ Department of Medicine, Bowen University Teaching Hospital, Ogbomoso, Nigeria \\ ${ }^{4}$ Department of Psychiatry, Lagos University Teaching Hospital, Lagos, Nigeria \\ Email: oniffpo@yahoo.com
}

Received 25 November 2013; revised 20 December 2013; accepted 27 December 2013

Copyright (C) 2014 Peter Olutunde Onifade et al. This is an open access article distributed under the Creative Commons Attribution License, which permits unrestricted use, distribution, and reproduction in any medium, provided the original work is properly cited. In accordance of the Creative Commons Attribution License all Copyrights (C) 2014 are reserved for SCIRP and the owner of the intellectual property Peter Olutunde Onifade et al. All Copyright (C) 2014 are guarded by law and by SCIRP as a guardian.

\section{ABSTRACT}

Background: Use of alcohol and other psychoactive substances constitutes major public health concern, especially among adolescents and young adults. The aims of this study were to determine the prevalence and perceived accessibility of psychoactive substances. Method: This cross sectional study was conducted in three Nigerian universities with the use of United Nations Office on Drugs and Crime (UNODC) student model questionnaire. Results: Five hundred and forty-nine students participated. Majority of them were females, 289 (55.2\%); Christians, 382 (73.0\%); of Yoruba ethnic group, 375 (79.6\%), and single, 512 $(94.8 \%)$. Their mean age was 20.11 years $(s d=2.36)$. Stimulants other than the Amphetamine-types had the highest lifetime prevalence rate $(53.4 \%)$, followed by Alcohol $(35.8 \%)$, tranquilizers $(12 \%)$, opiates other than Heroin $(11.9 \%)$ and cigarettes $(11.3 \%)$. Among the illicit drugs, marijuana had the highest prevalence $(7.2 \%)$. Lifetime prevalence rates of cocaine and heroin use were both $2.1 \%$. The average age at first use of any of the substances was between 10 and 17 years. The commonest consequences of alcohol use were engaged in sex regretted the next day (2.5\%) and engaged in sex without using condom (2.1\%); while the commonest consequences of drug use were damages to objects or clothing $(\mathbf{1 . 1 \% )}$, problems in relationship with parents $\mathbf{( 1 . 0 \% )}$ and friends $(\mathbf{1 . 0 \% )}$. Stimulants other than amphetamine-types, solvents, opiates other than heroin, tranquilizers and

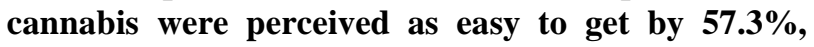
$38.7 \%, 32.8 \%, 29.4 \%$ and $22.7 \%$ of the respondents respectively. For all the substances except Ampheta- mine-type stimulants, ecstasy and crack, lifetime drug use was significantly associated with perceived easy accessibility to the respective substances. Conclusions and Recommendations: Drug use among the students was associated with relationship problems and unsafe sex. It is also associated with perceived accessibility of the drugs. Attention needs to be focused on safe sex practices among the students in addition to drug use prevention interventions.

\section{KEYWORDS}

Psychoactive Substance; Availability; University Students; Nigeria

\section{INTRODUCTION}

Alcohol use, unsafe sex and illicit drug use respectively contribute $8 \%, 5 \%$ and $2 \%$ of disability-adjusted lifeyears (DALYs) global burden of diseases among the 15 24-year-old adolescents. The health burden of alcohol and drug use is enormous. Among young people aged 10 - 24 years worldwide in 2004, alcohol use caused the loss of 71 million years of full health as measured by disability-adjusted life-years (DALYs). Only road traffic accidents and psychotic mental illness contributed greater health burden among this age group. Among males aged 15 - 19 years, 11 million healthy lives were lost due to misuse of psychoactive substances aside alcohol [1].

Among university students in Nigeria, the lifetime and one-month rates of the use of any psychoactive substance were reported to be $56 \%$ - $68 \%$ and $28 \%$ - $40 \%$ respectively [2,3]. The rate of lifetime alcohol use among un- 
dergraduates ranged between $1 \%$ and $85 \%$, [2-10]. The one-year range was $26 \%-62 \%$ [6,7]. One-month rate of alcohol use was $0.7 \%$ - 78\% [2-5,7-9]. Rate of lifetime cannabis use among them was $0.1 \%$ in the North-West zone of the country [8], 2.3\% in the North-Central [3] and ranged between 20\% [7] and 44\% [10] in the South-South geopolitical zone of the country. One-month rate of cannabis use was $0.6 \%-3.3 \%[3,7,8,11]$. The highest lifetime rate (16\%) of cocaine use was reported among undergraduates in the South-South [10]. In the 1999 United Nations International Drug Control Programme (UNDCP) survey, 1\% of tertiary school students abused cocaine [12]. For many undergraduates, use of psychoactive substances began in secondary schools. Among secondary school students aged 11 - 19 years in the South-East, the rate of use of any psychoactive substance was 63\% lifetime and 34\% one-month [13]. Among those aged 13 - 20 years in the North-Central, the rate was $91 \%$ lifetime and $72 \%$ one-month [14].

Many studies in Nigeria have reported the prevalence of substance use among university students, but there is paucity of data on the consequences and perceived accessibility of the substances around the university premises. The aims of this study were to determine the prevalence rate of substance use and consequences, and perceive accessibility of the substances.

\section{METHOD}

This cross sectional study was conducted in 2009-2010 in three Nigerian universities. Two of them were religion-based-one a Muslim (UNV A) and the other a Christian (UNV B). The third university (UNV C) was secular and owned by the Federal Government. The study was part of a larger study on social capital in the hostels of the three universities; so residence in the hostels was an inclusion criterion. Sampling was done proportionate to the size of each hostel. Systematic random technique was used to select the rooms. Every member of the selected rooms was selected for the study.

The United Nations Office on Drugs and Crime (UNODC) student model questionnaire was adapted for the study. The questionnaire is a 20-item instrument containing socio-demographic questions (items 1 - 6), lifetime, last 12-month and last 30-day substance use (items 7 - 14), age at first use of the substances (item 15), disapproval of the use of the substances (item 17), perceived risk of harm from substance use (item 18), perceived ease of accessibility to the substances (item 19) and personal experience of harm resulting from the use of alcohol, drugs or other causes [15]. The self administered questionnaire is reliable for drug use survey in $\mathrm{Ni}$ geria [16]. The questionnaire with the informed consent page was delivered in envelope to each selected student. The students were requested to enclose the filled ques- tionnaires in the envelopes and submit them to designated persons in the hostels. Values were not computed for missing data except in case of lifetime and 12-month drug use; for example, if lifetime response was blank, but 12 month was positive, lifetime was computed to be positive. The analysis was done with Statistical Package for Social Sciences (SPSS) version 16. Frequency, mean, median, mode and standard deviation were used for descriptive exploration. Chi-squared statistic was used to test association among categorical variables with p-value $<0.05$ taken as significant. In addition to ethical approval by the Research Ethics committee, permission letter was obtained from each of the three universities.

\section{RESULTS}

\subsection{Response Rate}

One hundred and twenty-three (125) questionnaires were given out in UNV A, 300 in UNV B and 250 in UNV C while 97, 254 and 198 filled questionnaire were returned from the three universities respectively, giving response rates of $77.6 \%, 84.6 \%$ and $79.2 \%$ respectively.

\subsection{Socio-Demographic Variables}

Table 1 shows the socio demographic variables of the universities. Majority of the participants were females, 289 (55.2\%); Christians, 382 (73.0); Yoruba, 375 (79.6\%), and single, 512 (94.8\%). As shown in Table 2,

Table 1. Socio-demographic variables.

\begin{tabular}{ccccc}
\hline Variable & Total (\%) & UNV A (\%) & UNV B (\%) & UNV C (\%) \\
\hline Respondents & $549(100.0)$ & $97(17.7)$ & $254(46.3)$ & $198(36.1)$ \\
Gender & & & & \\
Male & $235(44.8)$ & $31(33.7)$ & $116(48.1)$ & $88(46.1)$ \\
Female & $289(55.2)$ & $61(66.3)$ & $125(51.9)$ & $103(53.9)$ \\
Religion* $^{*}$ & & & & \\
$\quad$ Islam & $143(27.0)$ & $92(97.9)$ & $20(8.2)$ & $31(16.1)$ \\
Christianity $^{3}$ & $382(73.0)$ & $2(2.1)$ & $223(91.8)$ & $161(83.9)$ \\
Ethnicity & & & & \\
Yoruba & $375(79.6)$ & $77(95.1)$ & $146(67.6)$ & $152(87.4)$ \\
Others & $96(20.4)$ & $4(4.9)$ & $70(32.4)$ & $22(12.6)$ \\
Marital status & & & & \\
Single & $515(94.8)$ & $92(94.8)$ & $233(91.7)$ & $190(99.0)$ \\
Others & $28(5.2)$ & $5(5.2)$ & $21(8.3)$ & $2(1.0)$ \\
\hline
\end{tabular}

${ }^{*}$ p-value $<0.05$.

Table 2. Mean age of respondents.

\begin{tabular}{cccccc}
\hline & n & Mean (95 CI) & sd & \multicolumn{2}{c}{ ANOVA } \\
& & & & F & p \\
\hline UNV A & 85 & $19.93(19.43-20.43)$ & 2.298 & & \\
UNV B & 219 & $20.30(19.97-20.63)$ & 2.477 & 1.251 & 0.287 \\
UNV C & 162 & $19.96(19.62-20.31)$ & 2.220 & & \\
Total & 466 & $20.11(19.90-20.33)$ & 2.360 & & \\
\hline
\end{tabular}


the mean age of the participants was 20.11 (sd $=2.36$ ) with no statistically significant difference among the universities $(\mathrm{F}=1.251, \mathrm{p}=0.287)$.

\subsection{Prevalence of Drug Use}

Lifetime prevalence of drug use is depicted in Table 3. Stimulants other than the Amphetamine-types had the highest lifetime prevalence rate (53.4\%), followed by Alcohol (35.8\%), other drugs like power horse (25.1\%), tranquilizers (12\%), opiates other than Heroin $(11.9 \%)$ and cigarettes (11.3\%). Among the illicit drugs, marijuana had the highest prevalence (7.2\%). Lifetime prevalence rates of cocaine and heroin use were both $2.1 \%$. Lifetime rates of use for some of the drugs (like cigarettes, alcohol, and marijuana) were significantly different among the three universities.

Table 3 also shows the 12-month prevalence rate of drug use. Stimulants other than the Amphetamine-types still had the highest one-year prevalence rate (40.2\%), followed by Alcohol (25.0\%), opiates other than Heroin (8.6\%), cigarettes (8.4\%) and tranquilizers (7.5\%). Among the illicit drugs, marijuana had the highest 12-month prevalence (5.0\%). Twelve-month prevalence rates of cocaine and heroin use were both $1.4 \%$.

Thirty-day prevalence of drug use is shown in Table 3. Stimulants other than the Amphetamine-types remained the substance with the highest 30-day prevalence rate (23.3\%), followed by Alcohol (13.3\%), solvents/ inhalants (4.7\%), cigarettes (3.7\%), marijuana (3.5\%) and opiates other than Heroin (3.5\%). Among the illicit drugs, marijuana had the highest one-year prevalence (5.0\%). 30 -day prevalence rates of cocaine and heroin use were both $1.2 \%$.

\subsection{Age at Onset of Drug Use}

Table 4 shows the age at first use of the substances. The average age at first use of any of the substances was between 10 and 17 years. By the age of between 15 and 19 years, $50 \%$ (50th percentile) of the students had used at least one of the substances.

\subsection{Consequences of Drug Use}

As seen in Table 5, the commonest consequences of alcohol use were engaging in sex regretted the next day (2.5\%), quarrel or argument (2.3\%), engaging in sex without using condom (2.1\%) and damage to objects or clothing (2.1\%). The commonest consequences of drug use were damage to objects or clothing (1.1\%), problems in relationship with parents (1.0\%) and friends (1.0\%).

\subsection{Perceived Drug Accessibility and Lifetime Substance Use}

Other drugs like power horse, stimulants other than am- phetamine-types, solvents, opiates other than heroin, tranquilizers and cannabis were perceived as easy to get by $58.1 \%, 57.3 \%, 38.7 \%, 32.8 \%, 29.4 \%$ and $22.7 \%$ of the respondents respectively (see Table 6).

Table 7 shows the association between lifetime substance use and perceived accessibility to the substance. For all the substances except Amphetamine-type stimulants, Ecstasy and crack, lifetime drug use was significantly associated with perceived easy accessibility to the respective substances at p-value less than 0.05 .

\section{DISCUSSION}

\subsection{Prevalence of Drug Use}

The two most commonly used substances at lifetime; 12-month and 30-day periods were stimulants other than Amphetamine-Type and alcohol. Prevalence rate of opiates other than heroin ranked among the first 5 at lifetime, 12-month and 30-day periods. Marijuana and inhalant/solvents were among the five most commonly used substances in 30-day, but not the lifetime and 12 month period. The 12-month and 30-day rates of cocaine and heroin use were nearly the same.

The prevalence rates of psychoactive substances among the students were comparable to rates reported in some secondary schools and other universities in Nigeria [17-19]. Rate of current use of cigarettes in this study was greater than the $0 \%$ and $0.1 \%$ reported among Nigerian household women aged 15 - 19 years and 20 - 24 years respectively. It about doubles the $1.4 \%$ reported among household men aged 15 - 19 years but lower than the $6 \%$ reported among 20 - 24 year-old men [20].

\subsection{Consequences of Drug Use}

While damage to objects or clothes, problems in relationship with parents, and friends were often associated with drug use, the most common consequences of alcohol use in this study were engaging in sex regretted the following day, quarrels, and engaging in sex without condom. In the Nigerian households, sex related alcohol use problems have been reported in which $68.4 \%$ of ever-married women aged 15 - 49 whose partners got drunk often experienced emotional, physical or sexual violence compared to women whose partners did not drink often experienced emotional, physical or sexual violence compared to women whose partners did not drink alcohol.

Similarly, $6.5 \%$ of women whose husbands got drunk often committed physical violence against their partners compared with $0.5 \%$ of women whose partners did not drink [20].

An incidental finding in this study was that dysfunctional relationship, physical injury and damage to objects or clothing were mostly due to reasons other than use of 
Table 3. Lifetime, 12-month and 30-day rates of psychoactive substance use.

\begin{tabular}{cccccccccc}
\hline & \multicolumn{3}{c}{ Lifetime } & & \multicolumn{3}{c}{ 12-month } & & 30-day \\
\hline & UNV A & UNV B & UNV C & UNV A & UNV B & UNV C & UNV A & UNV B & UNV C \\
\hline Cigarettes & $6(6.3)$ & $44(18.7)$ & $8(18.7)$ & $4(4.2)$ & $32(13.6)$ & $7(3.8)$ & $2(2.1)$ & $14(6.0)$ & $3(1.6)$ \\
Alcohol & $16(16.7)$ & $112(47.7)$ & $53(30.5)$ & $10(10.4)$ & $83(35.3)$ & $33(19.0)$ & $5(5.2)$ & $39(16.6)$ & $23(13.2)$ \\
Marijuana & $4(4.3)$ & $28(11.3)$ & $5(2.8)$ & $2(2.2)$ & $20(8.1)$ & $4(2.2)$ & $1(1.1)$ & $14(5.8)$ & $3(1.7)$ \\
Tranquillizers & $6(6.5)$ & $35(14.2)$ & $21(11.9)$ & $5(5.4)$ & $27(10.9)$ & $7(3.9)$ & $3(3.3)$ & $6(2.5)$ & $1(.6)$ \\
Amphetamines & $0(0.0)$ & $1(0.4)$ & $3(1.7)$ & $0(0.0)$ & $1(0.4)$ & $2(1.1)$ & $0(0.0)$ & $1(.4)$ & $1(.6)$ \\
Metamphetamines & $0(0.0)$ & $2(0.8)$ & $5(2.8)$ & & & & & & \\
Other stimulants & $52(56.5)$ & $151(61.1)$ & $73(41.0)$ & $43(46.7)$ & $108(43.7)$ & $57(32.0)$ & $24(26.1)$ & $57(23.3)$ & $38(21.8)$ \\
Ecstasy & $3(3.3)$ & $3(1.2)$ & $10(5.7)$ & $2(2.2)$ & $2(0.8)$ & $5(2.8)$ & $0(0.0)$ & $1(0.4)$ & $3(1.7)$ \\
Other hallucinogens & $2(2.2)$ & $6(2.5)$ & $4(2.3)$ & $2(2.2)$ & $5(2.0)$ & $2(1.1)$ & $2(2.2)$ & $3(1.2)$ & $2(1.1)$ \\
Cocaine & $4(4.3)$ & $5(2.0)$ & $2(1.1)$ & $2(2.2)$ & $4(1.6)$ & $1(0.6)$ & $1(1.1)$ & $4(1.7)$ & $1(0.6)$ \\
Crack & $1(1.1)$ & $4(1.6)$ & $3(1.7)$ & $0(0.0)$ & $4(1.6)$ & $2(1.1)$ & $0(0.0)$ & $2(0.8)$ & $1(0.6)$ \\
Heroin & $2(2.2)$ & $6(2.5)$ & $3(1.7)$ & $1(1.1)$ & $5(2.0)$ & $1(0.6)$ & $1(1.1)$ & $4(1.7)$ & $1(0.6)$ \\
Other opiates & $13(14.1)$ & $36(14.7)$ & $12(6.8)$ & $12(13.0)$ & $27(11.0)$ & $7(3.9)$ & $5(5.4)$ & $9(3.7)$ & $4(2.3)$ \\
Drugs by injection & $6(6.5)$ & $6(2.4)$ & $8(4.5)$ & $3(3.3)$ & $4(1.6)$ & $6(3.4)$ & $1(1.1)$ & $4(1.7)$ & $3(1.7)$ \\
Solvents/Inhalant & $11(12.0)$ & $21(8.5)$ & $17(9.6)$ & $8(8.7)$ & $14(5.7)$ & $14(7.9)$ & $5(5.5)$ & $10(4.1)$ & $9(5.2)$ \\
Analgesics & $54(58.7)$ & $125(50.8)$ & $88(49.4)$ & $46(50.0)$ & $97(39.4)$ & $63(35.2)$ & $33(36.3)$ & $65(26.7)$ & $39(22.3)$ \\
Other drugs & $28(30.4)$ & $66(28.2)$ & $31(18.0)$ & & & & & \\
\hline
\end{tabular}

Table 4. Ages at first use of the drugs.

\begin{tabular}{|c|c|c|c|c|c|c|}
\hline & \multirow[t]{2}{*}{$\mathbf{N}$} & \multirow[t]{2}{*}{ Mean } & \multirow[t]{2}{*}{ sd } & \multicolumn{3}{|c|}{ Percentiles } \\
\hline & & & & 25 & 50 & 75 \\
\hline Cigarettes & 63 & 15.98 & 3.661 & 14.00 & 17.00 & 19.00 \\
\hline Alcohol & 211 & 15.87 & 3.528 & 14.00 & 17.00 & 18.00 \\
\hline Marijuana & 30 & 17.07 & 2.852 & 15.75 & 17.00 & 19.00 \\
\hline Tranquillizers & 38 & 15.68 & 3.625 & 14.00 & 16.00 & 18.25 \\
\hline \multicolumn{7}{|l|}{ Amphetamines } \\
\hline Methamphetamine & 1 & 10.00 & 10 & 10.00 & 10.00 & \\
\hline Other stimulants & 151 & 14.22 & 3.946 & 10.00 & 15.00 & 17.00 \\
\hline Ecstasy & 2 & 16.50 & 2.121 & 15.00 & 16.50 & 18.00 \\
\hline \multicolumn{7}{|l|}{ LSD $^{*}$} \\
\hline Other hallucinogens & 6 & 13.33 & 5.610 & 7.50 & 14.00 & 18.50 \\
\hline Cocaine & 5 & 13.60 & 6.504 & 7.50 & 13.00 & 20.00 \\
\hline Crack & 3 & 16.67 & 4.041 & 12.00 & 19.00 & 19.00 \\
\hline Heroin & 6 & 16.50 & 3.082 & 14.00 & 17.50 & 19.00 \\
\hline Other opiates & 41 & 15.17 & 4.260 & 12.00 & 16.00 & 18.00 \\
\hline Drugs by injection & 9 & 14.44 & 5.548 & 11.00 & 14.00 & 19.50 \\
\hline Solvents/inhalants & 34 & 12.29 & 3.648 & 9.75 & 12.00 & 15.00 \\
\hline Analgesics & 171 & 12.96 & 4.298 & 10.00 & 13.00 & 16.00 \\
\hline Other drugs & 97 & 16.93 & 2.898 & 15.00 & 17.00 & 19.00 \\
\hline
\end{tabular}

*LSD-Lysergic acid diethylamide. 
Table 5. Sequels of substance use.

\begin{tabular}{ccccc}
\hline Consequence & Never & Due to alcohol use & Due to the use of other substances & Due to other reasons \\
\hline Quarrel & $102(21.3)$ & $11(2.3)$ & $4(0.8)$ & $362(75.6)$ \\
Scuffle & $271(57.1)$ & $4(0.8)$ & $2(0.4)$ & $198(41.7)$ \\
Accident & $234(49.0)$ & $4(0.8)$ & & $240(50.2)$ \\
Loss of money & $152(31.9)$ & $9(1.9)$ & $3(0.6)$ & $313(65.6)$ \\
Damage to objects & $246(52.2)$ & $10(2.1)$ & $5(1.1)$ & $210(44.6)$ \\
Problems with parents & $300(62.8)$ & $5(1.0)$ & $5(1.0)$ & $168(35.1)$ \\
Problems with friends & $235(49.2)$ & $10(1.0)$ & $5(1.0)$ & $228(47.7)$ \\
Problems with neigbours & $362(77.7)$ & $2(0.4)$ & $4(0.9)$ & $98(21.0)$ \\
Expelled from school & $460(96.4)$ & $4(0.8)$ & $2(0.4)$ & $11(2.3)$ \\
Poor school performance & $349(73.6)$ & $7(1.5)$ & $3(0.6)$ & $115(24.3)$ \\
Victimized by robbery or theft & $419(87.7)$ & $6(1.3)$ & & $53(11.1)$ \\
Trouble with police & $447(93.7)$ & $7(1.5)$ & $1(0.2)$ & $22(4.6)$ \\
Hospitalized & $412(86.2)$ & $2(0.4)$ & & $64(13.4)$ \\
Sex regretted the next day & $412(87.1)$ & $12(2.5)$ & $1(0.2)$ & $48(10.1)$ \\
Sex without using condom & $389(82.2)$ & $10(2.1)$ & $1(0.2)$ & $73(15.4)$ \\
\hline
\end{tabular}

Tables 6. Perceived accessibility to substances among the universities.

\begin{tabular}{|c|c|c|c|c|c|c|c|c|}
\hline \multicolumn{2}{|c|}{ Substance and ease of getting it } & \multirow{3}{*}{$\begin{array}{c}\text { Total (\%) } \\
285(77.2) \\
84(22.8)\end{array}$} & \multirow{3}{*}{$\begin{array}{c}\text { UNV A (\%) } \\
51 \text { (77.3) } \\
15(22.7)\end{array}$} & \multirow{3}{*}{$\begin{array}{c}\text { UNV B (\%) } \\
138(71.1) \\
56(28.9)\end{array}$} & \multirow{2}{*}{$\begin{array}{c}\text { UNV C (\%) } \\
96(88.1)\end{array}$} & \multirow{3}{*}{\begin{tabular}{c|}
$\mathbf{x}^{2}$ \\
11.39
\end{tabular}} & \multirow{3}{*}{$\begin{array}{c}\text { df } \\
2\end{array}$} & \multirow{3}{*}{$\begin{array}{c}\mathbf{p} \\
0.003\end{array}$} \\
\hline Marijuana & Difficult & & & & & & & \\
\hline & Easy & & & & $13(11.9)$ & & & \\
\hline \multirow[t]{2}{*}{ Tranquillizer } & Difficult & $263(72.1)$ & $48(70.6)$ & $129(67.2)$ & 86 (81.9) & \multirow{2}{*}{7.39} & \multirow{2}{*}{2} & \multirow{2}{*}{0.025} \\
\hline & Easy & $102(27.9)$ & $20(29.4)$ & $63(32.8)$ & $19(18.1)$ & & & \\
\hline \multirow[t]{2}{*}{ Amphetamines } & Difficult & 318 (94.1) & $61(96.8)$ & $158(91.3)$ & 99 (97.1) & & & \\
\hline & Easy & $20(5.9)$ & $2(3.2)$ & $15(8.7)$ & $3(2.9)$ & & & \\
\hline \multirow[t]{2}{*}{ Methamphetamine } & Difficult & $316(95.5)$ & $61(98.4)$ & $160(93.6)$ & 95 (96.9) & \multirow{2}{*}{3.14} & \multirow{2}{*}{2} & \multirow{2}{*}{0.208} \\
\hline & Easy & $15(4.5)$ & $1(1.6)$ & $11(6.4)$ & $3(3.1)$ & & & \\
\hline \multirow[t]{2}{*}{ Other stimulants } & Difficult & $172(44.4)$ & $32(42.7)$ & $74(36.3)$ & $66(61.1)$ & \multirow{2}{*}{17.76} & \multirow{2}{*}{2} & \multirow{2}{*}{$<0.000$} \\
\hline & Easy & 215 (55.6) & $43(57.3)$ & $130(63.7)$ & $42(38.9)$ & & & \\
\hline \multirow[t]{2}{*}{ Ecstasy } & Difficult & $298(92.0)$ & $58(96.7)$ & $150(88.2)$ & $90(95.7)$ & \multirow{2}{*}{6.82} & \multirow{2}{*}{2} & \multirow{2}{*}{0.033} \\
\hline & Easy & $26(8.0)$ & $2(3.3)$ & $20(11.8)$ & $4(4.3)$ & & & \\
\hline \multirow[t]{2}{*}{ LSD } & Difficult & $310(95.7)$ & 58 (96.7) & $159(93.0)$ & $93(100.0)$ & \multirow{2}{*}{7.34} & \multirow{2}{*}{2} & 0025 \\
\hline & Easy & $14(4.3)$ & $2(3.3)$ & $12(7.0)$ & $0(.0)$ & & & 0.025 \\
\hline Other hallucinogens & Difficult & $304(92.4)$ & 57 (91.9) & $154(90.6)$ & 93 (95.9) & 248 & 2 & 0289 \\
\hline & Easy & $25(7.6)$ & $5(8.1)$ & $16(9.4)$ & $4(4.1)$ & 2.40 & 2 & 0.203 \\
\hline Cocaine & Difficult & 325 (93.4) & 61 (95.3) & $165(89.7)$ & 99 (99.0) & 959 & 2 & 0008 \\
\hline & Easy & $23(6.6)$ & $3(4.7)$ & $19(10.3)$ & $1(1.0)$ & 5.03 & 2 & 0.000 \\
\hline Crack & Difficult & 323 (95.6) & 59 (98.3) & 167 (92.8) & 97 (99.0) & 708 & 2 & 0.029 \\
\hline & Easy & $15(4.4)$ & $1(1.7)$ & $13(7.2)$ & $1(1.0)$ & 1.08 & 2 & 0.029 \\
\hline Heroin & Difficult & 317 (92.4) & 57 (91.9) & $165(90.7)$ & 95 (96.0) & 259 & 2 & \\
\hline & Easy & $26(7.6)$ & $5(8.1)$ & $17(9.3)$ & $4(4.0)$ & 2.59 & 2 & 0.270 \\
\hline Other opiates & Difficult & $265(75.1)$ & $43(67.2)$ & $130(69.5)$ & $92(90.2)$ & 17.67 & 2 & $<0.001$ \\
\hline & Easy & 88 (24.9) & $21(32.8)$ & 57 (30.5) & $10(9.8)$ & & & \\
\hline Drugs by injection & Difficult & $316(90.0)$ & $61(92.4)$ & 159 (87.8) & 96 (92.3) & 198 & 2 & 0371 \\
\hline & Easy & $35(10.0)$ & $5(7.6)$ & $22(12.2)$ & $8(7.7)$ & 1.50 & 2 & $0.5 / 1$ \\
\hline Solvents/inhalants & Difficult & 225 (59.4) & $46(61.3)$ & $108(54.5)$ & $71(67.0)$ & 4.57 & 2 & 0.101 \\
\hline & Easy & $154(40.6)$ & 29 (38.7) & $90(45.5)$ & 35 (33.0) & 4.57 & 2 & 0.101 \\
\hline Analgesics & Difficult & $142(36.0)$ & 22 (28.9) & 64 (31.2) & $56(49.6)$ & & & \\
\hline & Easy & $252(64.0)$ & $54(71.1)$ & 141 (68.8) & $57(50.4)$ & 12.68 & 2 & 0.002 \\
\hline Other drugs & Difficult & $202(53.2)$ & 31 (41.9) & $98(49.5)$ & 73 (67.6) & 1387 & 2 & 0001 \\
\hline & Easy & 178 (46.8) & $43(58.1)$ & $100(50.5)$ & 35 (32.4) & $10.0 \%$ & 2 & 0.001 \\
\hline
\end{tabular}


Tables 7. Perceived substance accessibility and lifetime uses.

\begin{tabular}{|c|c|c|c|c|c|c|c|}
\hline \multirow[b]{2}{*}{ Substance } & \multirow[b]{2}{*}{ Perceived accessibility } & \multicolumn{5}{|c|}{ Lifetime use } & \multirow[b]{2}{*}{ p } \\
\hline & & Total & No & Yes & $x^{2}$ & df & \\
\hline \multirow[t]{3}{*}{ Marijuana } & Difficult to get & $278(76.8)$ & $276(83.1)$ & $2(6.7)$ & \multirow{2}{*}{90.28} & \multirow{2}{*}{1} & \multirow{2}{*}{$<0.001$} \\
\hline & Easy to get & $84(23.2)$ & $56(16.9)$ & $28(93.3)$ & & & \\
\hline & Total & $362(100.0)$ & $332(100.0)$ & $30(100.0)$ & & & \\
\hline \multirow[t]{3}{*}{ Tranquillizer } & Difficult to get & 257 (71.6) & $240(76.9)$ & $17(36.2)$ & \multirow{3}{*}{33.35} & \multirow{3}{*}{1} & \multirow{3}{*}{$<0.001$} \\
\hline & Easy to get & $102(28.4)$ & $72(23.1)$ & $30(63.8)$ & & & \\
\hline & Total & $359(100.0)$ & $312(100.0)$ & $47(100.0)$ & & & \\
\hline \multirow[t]{3}{*}{ Amphetamines } & Difficult to get & $312(94.0)$ & $311(94.0)$ & $1(100.0)$ & \multirow{2}{*}{0.06} & \multirow{2}{*}{1} & \multirow{2}{*}{0.800} \\
\hline & Easy to get & $20(6.0)$ & $20(6.0)$ & $0(0.0)$ & & & \\
\hline & Total & $332(100.0)$ & $331(100.0)$ & $1(100.0)$ & & & \\
\hline \multirow[t]{3}{*}{ Methamphetamine } & Difficult to get & 309 (95.4) & 306 (95.6) & $3(75.0)$ & \multirow{3}{*}{3.80} & \multirow{3}{*}{1} & \multirow{3}{*}{0.051} \\
\hline & Easy to get & $15(4.6)$ & $14(4.4)$ & $1(25.0)$ & & & \\
\hline & Total & $324(100.0)$ & $320(100.0)$ & $4(100.0)$ & & & \\
\hline Other stimulants & Difficult to get & $168(44.0)$ & $112(67.5)$ & $56(25.9)$ & 6575 & 1 & $<0001$ \\
\hline & Easy to get & $214(56.0)$ & $54(32.5)$ & $160(74.1)$ & $03 . / 5$ & 1 & $<0.001$ \\
\hline & Total & $382(100.0)$ & $166(100.0)$ & $216(100.0)$ & & & \\
\hline Ecstasy & Difficult to get & $290(91.8)$ & 281 (91.5) & $9(100.0)$ & 083 & 1 & 0362 \\
\hline & Easy to get & $26(8.2)$ & $26(8.5)$ & $0(0.0)$ & 0.03 & 1 & 0.002 \\
\hline & Total & $316(100.0)$ & $307(100.0)$ & $9(100.0)$ & & & \\
\hline LSD & Difficult to get & 303 (95.6) & 302 (95.9) & $1(50.0)$ & 091 & 1 & בח00 \\
\hline & Easy to get & $14(4.4)$ & $13(4.1)$ & $1(50.0)$ & 9.91 & 1 & 0.002 \\
\hline & Total & $317(100.0)$ & $315(100.0)$ & $2(100.0)$ & & & \\
\hline Other hallucinogens & Difficult to get & 298 (92.3) & $296(93.1)$ & $2(40.0)$ & 19.42 & 1 & $<0.001$ \\
\hline & Easy to get & $25(7.7)$ & $22(6.9)$ & $3(60.0)$ & & & \\
\hline & Total & $323(100.0)$ & $318(100)$ & $5(100.0)$ & & & \\
\hline Cocaine & Difficult to get & 318 (93.3) & 315 (94.3) & $3(42.9)$ & 2126 & 1 & $<0 \Omega 01$ \\
\hline & Easy to get & $23(6.7)$ & $19(5.7)$ & $4(57.1)$ & 21.20 & 1 & 20.001 \\
\hline & Total & $341(100.0)$ & $334(100.0)$ & $7(100.0)$ & & & \\
\hline Crack & Difficult to get & 316 (95.5) & 312 (95.7) & $4(80.0)$ & 281 & 1 & 0094 \\
\hline & Easy to get & $15(4.5)$ & $14(4.3)$ & $1(20.0)$ & 2.01 & 1 & 0.094 \\
\hline & Total & $331(100.0)$ & $326(100.0)$ & $5(100.0)$ & & & \\
\hline Heroin & Difficult to get & 311 (92.3) & 308 (93.1) & $3(50.0)$ & 1534 & 1 & $<0001$ \\
\hline & Easy to get & $26(7.7)$ & $23(6.9)$ & $3(50.0)$ & 10.04 & 1 & 0.001 \\
\hline & Total & $337(100.0)$ & $331(100.0)$ & $6(100.0)$ & & & \\
\hline Other opiates & Difficult to get & $260(74.7)$ & $249(82.5)$ & 11 (23.9) & 7240 & 1 & $<0001$ \\
\hline & Easy to get & $88(25.3)$ & $53(17.5)$ & 35 (76.1) & 12.40 & 1 & $<0.001$ \\
\hline & Total & $348(100.0)$ & $302(100.0)$ & $46(100.0)$ & & & \\
\hline Drugs by injection & Difficult to get & 311 (89.9) & 306 (91.9) & $5(38.5)$ & 39.28 & 1 & $<0.001$ \\
\hline & Easy to get & $35(10.1)$ & $27(8.1)$ & $8(61.5)$ & & & \\
\hline & Total & $346(100.0)$ & $333(100.0)$ & $13(100.0)$ & & & \\
\hline Solvents/Inhalants & Difficult to get & $221(59.2)$ & $213(62.8)$ & $8(23.5)$ & 1977 & 1 & $<0001$ \\
\hline & Easy to get & $152(40.8)$ & $126(37.2)$ & $26(76.5)$ & 19.11 & 1 & 0.001 \\
\hline & Total & 373 (100.0) & 339 (100.0) & $34(100.0)$ & & & \\
\hline Analgesics & Difficult to get & 138 (35.7) & $112(64.4)$ & $26(12.2)$ & 113 & 1 & $<0,001$ \\
\hline & Easy to get & 249 (64.3) & $62(35.6)$ & $187(87.8)$ & 1.13 & 1 & $<0.001$ \\
\hline & Total & 387 (100.0) & $174(100.0)$ & $213(100.0)$ & & & \\
\hline Other drugs & Difficult to get & $188(52.1)$ & 179 (69.1) & $9(8.8)$ & 106 & 1 & $<0001$ \\
\hline & Easy to get & 173 (47.9) & 80 (30.9) & 93 (91.2) & 1.00 & 1 & 0.001 \\
\hline & Total & $361(100.0)$ & $259(100.0)$ & $102(100.0)$ & & & \\
\hline
\end{tabular}


alcohol and other substances. Of great public health concern is that, for reasons other than alcohol and drug use, 1 out of every 10 students engaged in sex regretted the next day and that 3 out of every 20 engaged in sex without using condom.

\subsection{Perceived Drug Accessibility and Lifetime Substance Use}

The significant association between a perceived accessibility and lifetime use of the substance can have direction in both ways. This study is unable to provide information on the direction of causality.

\subsection{Strengths}

This study reported not only the prevalence rate of substance use, but also the perceived accessibility and experience of personal harm from the substance use. It also provides data to compare substance use in three Nigerian Universities with different religious orientations.

\subsection{Limitations}

The study population was located in the South-Western part of Nigeria. Therefore its results may not be generalizable over the entire country. The study assessed consequences of substance use but did not explore the reasons why these students used the drugs they use.

\section{CONCLUSION AND RECOMMENDATIONS}

Drug use among the students was associated with physical injury, relationship problems, and unsafe sex. It was associated with perceived accessibility of the drugs.

While programs are needed to address drug use and its negative consequences, attention also needs to be focused on the safe sex practices among the students.

Further study is needed to determine the direction of causality between drug use and perceived accessibility of the drug.

\section{ACKNOWLEDGEMENTS}

Thanks to the authorities of the three universities for the permission to conduct the study.

\section{REFERENCES}

[1] Gore, F.M., Bloem, P.J., Patton, G.C., Ferguson, J., Joseph. V., Coffey, C., et al. (2011) Global burden of disease in young people aged 10 - 24 years: A systematic analysis. Lancet, 377, 2093-2102. http://dx.doi.org/10.1016/S0140-6736(11)60512-6

[2] Ihezue, U.H. (1988) Drug abuse among medical students at a Nigerian university: Part 1 . Prevalence and pattern of use. Journal of the National Medical Association, 80, 81-85.

[3] Makanjuola, A.B., Daramola, T.O. and Obembe, A.O. (2007) Psychoactive substance use among medical students in a Nigerian university. World Psychiatry, 6, 112114.

[4] Chikere, E.I. and Mayowa, M.O. (2011) Prevalence and perceived health effect of alcohol use among male undergraduate students in Owerri, South-East Nigeria: A descriptive cross-sectional study. BMC Public Health, 11, 118. http://dx.doi.org/10.1186/1471-2458-11-118

[5] Adewuya, A.O. (2005) Validation of the alcohol use disorders identification test (audit) as a screening tool for alcohol-related problems among Nigerian university students. Alcohol, 40, 575-577.

[6] Adewuya, A.O. (2006) Prevalence of major depressive disorder in Nigerian college students with alcohol-related problems. General Hospital Psychiatry, 28, 169-173. http://dx.doi.org/10.1016/j.genhosppsych.2005.09.002

[7] Nevadomsky, J.J. (1985) Drug use among Nigerian university students: Prevalence of self-reported use and attitudes to use. Bulletin on Narcotics, 37, 31-42.

[8] Yunusa, M.A., Obembe, A., Madawaki, A. and Asogwa, F. (2011) A survey of psychostimulants use among a university students in Northwestern Nigeria. Nigerian Journal of Psychiatry, 9, 40-45.

[9] Achalu, O.E. and D.F. (1987) Duncan, drug-taking among Nigerian students at universities in the United States of America. Bulletin on Narcotics, 39, 75-80.

[10] Okoza, J., Aluede, O. Fajoju, S. and Okhiku, I. (2009) Drug abuse among students of Ambrose Alli University, Ekpoma, Nigeria. European Journal of Social Sciences, 10, 85-92.

[11] Ihezue, U.H. (1988) Alcohol and drug taking among medical students at a Nigerian university campus: Part 2. Sociodemographic factors of etiologic significance. Journal of the National Medical Association, 80, 191-195.

[12] UNDCP (1999) Report of the rapid situation assessment of drug abuse in Nigeria. United Nations International Drug Control Programme, Nigeria.

[13] Igwe, W.C. and Ojinnaka N.C. (2010) Mental health of adolescents who abuse psychoactive substances in Enugu, Nigeria-A cross-sectional study. Italian Journal of Pediatrics, 36, 53.

[14] Adelekan, M.L. (1989) Self-reported drug use among secondary school students in the Nigerian state of Ogun. Bulletin on Narcotics, 41, 109-116.

[15] UNODC (2003) GAP toolkit module 3: Conducting school surveys on drug abuse. UN, New York

[16] Adelekan, M.L. and Odejide, O.A. (1989) The reliability and validity of the WHO student drug-use questionnaire among Nigerian students. Drug and Alcohol Dependence, 24, 245-249. http://dx.doi.org/10.1016/0376-8716(89)90062-8

[17] Oshodi, O.Y., Aina, O.F. and Onajole A.T. (2010) Substance use among secondary school students in an urban setting in Nigeria: Prevalence and associated factors. Afri- 
can Journal of Psychiatry (Johannesbg), 13, 52-57.

[18] Yisa, I.O., Lawoyin, T.O., Fatiregun, A.A. and Emelumadu, O.F. (2009) Pattern of substance use among senior students of command secondary schools in Ibadan, Nigeria. Nigerian Journal of Medicine, 18, 286-290.

[19] Fatoye, F.O. (2007) Substance use among university un- dergraduates: A study of pattern and beliefs in Ile-Ife. Nigerian Postgraduate Medical Journal, 14, 37-41.

[20] NPC and ICF (2009) Nigeria demographic and health survey. National Population Commission and ICF Macro, Abuja. 